\title{
1 Directed evolution of Vibrio fischeri LuxR signal sensitivity
}

2 Yuki Kimura, Yohei Tashiro, Kyoichi Saito, Shigeko, Kawai-Noma, and Daisuke Umeno*

3

4 Department of Applied Chemistry and Biotechnology, Chiba University, Chiba, JAPAN

5 1-33 Yayoi-cho, Inage-ku, Chiba City, Chiba 263-8522 JAPAN

$6 \quad$ E-mail address: umeno@faculty.chiba-u.jp

10 Running title: LuxR sensitivity mutant

11 Keywords: positive selection, genetic switch, quorum sensing, synthetic biology, chemical

12 chaperone, antagonist

13 


\section{Abstract}

2 LuxR is the core component of Vibrio fischeri quorum sensing. It acts as the

3 transcriptional activator by binding to its cognate signaling molecules 3-oxo-

4 hexanoyl-homoserine lactone (30C6HSL). Although several acyl-HSLs with 3-oxo

5 groups are known to activate LuxR with similar efficiency, acyl-HSLs without 3-oxo

6 groups are very weak inducers. We conducted a round of LuxR directed evolution to

7 acquire LuxR mutants with higher signal sensitivity to octanoyl-homoserine lactone

8 (C8HSL). All of the isolated mutants showed increased signal sensitivity to many

9 acyl-HSLs, including C8HSL, and some to the LuxR antagonist p-coumaroyl-HSL.

10 The evolution of ligand sensitivity proceeded through the stabilization of the signal-

11 bound state, thereby elevating the effective concentration of LuxR at the ON-state. 
1

2 Quorum sensing of Gram-negative bacteria is mainly mediated by acyl-homoserine

3 lactones (acyl-HSLs), which serve as signaling molecules (1). Diverse structural variation is

4 observed in acyl-HSLs that are biosynthesized in nature, and each acyl-HSL is synthesized

5 with the aim of activating/deactivating a cognate sensory protein. To clarify how this

6 remarkable diversity has arisen and to provide bacterial sensors (2) and cell-cell

7 communication devices $(3,4,5,6,7)$ to the synthetic biology community, quorum sensing

8 proteins have been extensively targeted for mutagenesis $(4,8,9)$.

9 The Vibrio fischeri Luxl/LuxR system is one of the most well-studied quorum sensing

10 systems among Gram-negative bacteria. In this system, 3-oxohexanoyl-HSL (3OC6HSL) is

11 synthesized by the enzyme Luxl, transported across the bacterial cell wall, and then

12 recognized by the sensor protein LuxR in both its source and other bacteria. Upon binding

13 to acyl-HSLs, LuxR undergoes a structural transition into the active dimer form and binds to

14 a specific DNA sequence called the lux box (10), thereby mediating the expression of the

15 downstream genes.

16 The functional plasticity (or evolvability) of LuxR has been explored extensively. Based on

17 structural modeling using the X-ray structure of TraR from Agrobacterium tumefaciens (11,

18 12), several residues have been shown to strongly influence the signal specificity of LuxR

19 (13). Arnold and her colleagues performed a directed evolution of $\operatorname{LuxR}(4,8,9)$ and 
1 different sets of amino acid substitutions that alter its signal specificity and sensitivity. For

2 instance, HSLs without 3-oxo groups are weak ligands for LuxR and thus require a high

3 concentration $(\sim 10 \mu \mathrm{M})$. Two rounds of mutagenesis followed by a screening search for

4 mutants expressing higher fluorescent marker levels (green fluorescent protein, GFP)

5 revealed that amino acid substitutions at positions $33,45,95,116,135$ and 174 , especially

6 combination, conferred elevated sensitivity to octanoyl-HSL (C8HSL) $(4,8,9)$.

7 In this work, we conducted a very similar directed evolution experiment of LuxR to

8 achieve higher sensitivity to C8HSL. For this purpose, we adopted a new selection system,

9 which we originally developed for the directed evolution of genetic circuits (14). In this

10 method, the entire selection process occurs in liquid, thus allowing many sets of different

11 selection programs to be conducted simultaneously. Using this method, we isolated

$12 \mathrm{C} 8 \mathrm{HSL}-$ sensitive LuxR mutants. An analysis of the isolated variants revealed that they were

13 sensitized to all of the tested HSLs (C6HSL, C8HSL, 30C6HSL, and 30C8HSL), which

14 suggests that there was an improvement in ligand sensitivity that proceeded through the

15 stabilization of the signal-bound state, thereby elevating the effective concentration of the

16 effectors in the ON-state. This "quick-and-dirty" solution may be the prevailing source of

17 novel LuxR family protein functions, possibly due to the rapid evolution of quorum-sensing

18 systems both in nature and in laboratories. 


\section{Materials and Methods}

\section{Bacterial strains, media, and growth conditions}

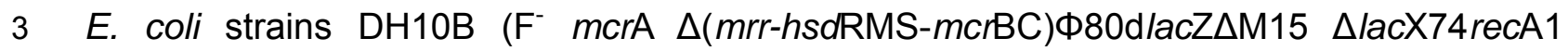

4 endA1 araD139 $\Delta($ ara, leu $) 7697$ galU ga/K $\lambda^{-} \operatorname{rpsL}\left(\mathrm{Str}^{\mathrm{R}}\right)$ nupG) (Invitrogen, CA) and XL10

5 Gold $\left(\right.$ Tet $^{\mathrm{R}} \Delta($ mcrA $) 183 \Delta($ mcrCB-hsdSMR-mrr)173 endA1 supE44 thi-1 recA1 gyrA96 relA1

6 lac Hte [F'proAB laclqZAM15 $\operatorname{Tn} 10\left(\operatorname{Tet}^{\mathrm{R}}\right) \operatorname{Tn} 5 \mathrm{Kan}^{\mathrm{R}}$ Amy]) (Stratagene, CA) were used for

7 cloning and library construction. $\Delta t d k$ strain JW1226 (F' $\Delta($ araD-araB)567 $\Delta$ lacZ4787(::rrnB-

8 3) $\lambda^{-} \Delta t d k-747:: k a n$ rph-1 $\Delta($ rhaD-rhaB)568, hsdR514) from the KEIO collection (15),

9 harboring $\mathrm{pAC}(\mathrm{Plux})-g f p_{\mathrm{uv}}$ or $\mathrm{pAC}(\mathrm{Plux})-\mathrm{HSVtk}$, was used for genetic selection. E. coli

10 strains were incubated in LB medium (2.0\% (w/v) Lennox LB; Invitrogen) or on LB agar

11 plates $\left(2.0 \%(\mathrm{w} / \mathrm{v}) \mathrm{LB}, 1.5 \%(\mathrm{w} / \mathrm{v})\right.$ agar; Nacalai tesque, JP) at $37^{\circ} \mathrm{C}$. Antibiotics were added

12 at the following concentrations: $30 \mathrm{\mu g} \mathrm{ml}^{-1}$ chloramphenicol (Nacalai tesque) to maintain the

$13 \mathrm{pACYC}$-based vector and/or $50 \mu \mathrm{g} \mathrm{ml}^{-1}$ carbenicillin (Invitrogen) to maintain the pTrc-based

14 vector.

15 The acyl-HSLs used in this study were 30C6HSL, 3-oxooctanoyl-HSL (3OC8HSL),

16 hexanoyl-HSL (C6HSL), C8HSL, and p-Coumaroyl-HSL (pCHSL). All of these compounds

17 were purchased from Sigma-Aldrich (MO). These stock solutions (1-10 mM) were prepared

18 by dissolving appropriate amounts of the compounds in ethyl acetate (Nacalai tesque)

19 acidified with glacial acetic acid $\left(0.01 \%(\mathrm{v} / \mathrm{v})\right.$; Nacalai tesque) and were stored at $-20^{\circ} \mathrm{C}$. 
$1 p C H S L$ stock solutions were prepared with ethanol instead of ethyl acetate (1-10 mM). For

2 the liquid-phase experiments, we dispensed acyl-HSL stock solutions into autoclaved 96-

3 deep-well plates, evaporated the ethyl acetate at room temperature, and then added the

4 medium containing cells to the dried acyl-HSL.

6 Plasmid construction

7 The luxR reading frame was PCR-cloned into the Ncol/Hindlll site of $\mathrm{pTrc99A}$, resulting in

8 pTrc-luxR. Similarly, the reading frames of gfp $\mathrm{uv}$ from pGFPuv (Clontech, CA) and herpes

9 simplex virus thymidine kinase (HSVtk) (14) were PCR-cloned into the Ncol/Hindlll site of

10 pACYC184 (p15A-ori, Chroramphenicol resistance $\left(\mathrm{Cm}^{\mathrm{R}}\right)$. The resultant pAC-gfpuv and

11 pAC-HSVtk were then PCR-cloned into Clal/Hindlll, yielding pAC(Ptrc)-gfpuv and

$12 \mathrm{pAC}(\mathrm{Ptrc})-\mathrm{HSV} t$, respectively. Next, the trc promoters of these plasmids were replaced

13 with the lux promoter

14 ACCTGTAGgATCGTACAGGTTTACGCAAGAAAATGGTTTGTTATAGTCGAATAAA-3'),

15 resulting in pAC(P/ux)-gfpuv and pAC(Plux)-HSVtk. The sequences of all of the above

16 plasmids were confirmed using the Big Dye ${ }^{\circledR}$ Terminator v3.1 Cycle Sequencing Kit (Life

17 Technologies Corporation, CA) and an ABI Prism 3100 Genetic Analyzer (Life Technologies

18 Corporation). 
1

2 Whole-gene mutagenesis was performed using pTrc-luxR (1 ng) as a template and a $1 \mu \mathrm{M}$

3 concentration of each primer (primer 1: 5'-CAATCTGTGTGGGCACTCGAC-3' and primer 2:

4 5'-TACTGCCGCCAGGCAAATTC-3'). The reaction mixtures (50 $\mu$ l total volume) contained

$55 \mathrm{U}$ of Taq DNA polymerase (New England Biolabs, MA), $200 \mu \mathrm{M}$ of each deoxynucleoside

6 triphosphate, $2 \mathrm{mM} \mathrm{MgCl}$, and $10 \mu \mathrm{M} \mathrm{MnCl}$, as described previously (16). The

7 amplification factor was approximately 1,000 (ca. 10 effective rounds). The PCR product

8 was subcloned into the Ncol/Hindlll site of pTrc99A. The ligation mixture was transformed

9 into Electro MAX DH10B by electroporation. DNA from the transformant was isolated by

10 miniprep to yield the library plasmid pTrc-[luxR] (library size $\approx 10^{6}$ ).

11

13 The luxR library was introduced into JW1226 harboring pAC(Plux)-HSVtk, and the cells

14 cultured overnight in $10 \mathrm{ml}$ of LB medium containing the appropriate type and concentration

15 of acyl-HSLs (in this case, $100 \mathrm{nM}$ of C8HSL). The transformants $\left(\approx 10^{6}\right.$ cells) from the

16 were inoculated into $\mathrm{ON}$-selection medium $(2.0 \%(\mathrm{w} / \mathrm{v})$ tryptone; Becton, Dickinson and

17 Company, MD, 0.5\%(w/v) NaCl; Nacalai tesque, $10 \mu \mathrm{g} \mathrm{ml}^{-1}$ thymidine; Wako, JP, $1 \mu \mathrm{g} \mathrm{ml}{ }^{-1}$

18 adenosine; Tokyo Chemical Industry Co., LTD., JP, and $20 \mu \mathrm{g} \mathrm{ml}^{-1} 2^{\prime}$-deoxy-5-fluorouridine

19 (5FdU); Sigma-Aldrich) and incubated for $20 \mathrm{~h}$. 5FdU is a potent inhibitor of thymidine 
1 synthase, in the presence of which cells fail to biosynthesize dTMP, a precursor of genomic

2 DNA. In this situation, only those cells expressing HSVTK can grow due to the capability of

3 HSVTK to phosphorylate exogenously added thymidine. From the pool of these "ON-

4 cells, plasmid DNA was isolated.

$6 \quad$ Site-directed mutagenesis

7 Using the ExSite method (Stratagene), PCR-based site-saturation or substitution

8 mutagenesis was performed on the pTrc-luxR template (10-20 ng) using an appropriate

9 primer set with altered codon sequences for the targeted site. The PCR fragments were

10 subcloned into the vector by using self-ligation. The ligation products were transformed into

11 XL10 Gold cells, plated and grown overnight on LB agar plates to isolate clones.

12 Similarly, site-saturation mutagenesis was induced for cysteine at amino acid position 245

13 (C245) using ExSite PCR with the primers containing NNK sequence ( $\mathrm{N}=$ equimolar

14 mixture of dATP, dCTP, dGTP, and dTTP; $\mathrm{K}$ = equimolar mixture of dGTP and dTTP) at the

15 targeted sites. The resultant plasmids were transformed into $\mathrm{DH} 10 \mathrm{~B}$. The transformants

16 were plated on LB agar plates. Approximately $10^{3}$ colonies from each plate were pooled,

17 and their plasmid DNA was isolated for subsequent analysis.

18

19

GFP-reporter analysis of gene expression 
1 For rapid screening, the library plasmids were transformed into JW1226 containing

$2 \operatorname{pAC}(\mathrm{Plux})$-gfpuv and incubated on LB agar plates overnight. These colonies were picked

3 and inoculated into $500 \mu \mathrm{L}$ of LB medium in 96-well deep-well plates. The cultures were

4 spotted with a 96-well pin replicator onto LB agar plates containing acyl-HSL. After 12-20 h,

5 LuxR mutants with the desired phenotype were visually selected under $365 \mathrm{~nm}$ UV light.

6 For the quantitative assay, JW1226 harboring $\mathrm{pAC}(\mathrm{Plux})$-gfp $\mathrm{Uv}$ and plasmids encoding

7 variant LuxR genes were first grown overnight from single colonies or glycerol stocks in LB

8 medium, and then the cultures were diluted 1,000-fold into $500 \mu \mathrm{L}$ of fresh LB medium

9 containing appropriate antibiotics and acyl-HSLs in 96-well deep-well plates (Qiagen, CA).

10 These cultures were shaken for $12 \mathrm{~h}$. The cultures $(20 \mu \mathrm{l}$ each) were diluted 10 -fold into

11 fresh LB medium (a total volume of $200 \mu \mathrm{l}$ ) in 96-micro-well plates (Nacalai tesque). GFPuv

12 fluorescence (390 nm excitation, $510 \mathrm{~nm}$ emission) was measured using a fluorescence

13 microplate reader (Thermo Fisher Scientific Inc., FLUOROSKAN ASCENT); cell densities

14 were measured using a microplate reader (Molecular Devices, SpectraMax Plus 384) at

$15600 \mathrm{~nm}$. The fluorescence of the cell suspensions was normalized to their optical densities.

17 Western blot analysis of LuxR variants

18 A histidine hexamer was fused in frame to the $\mathrm{N}$-terminus of the reading frames of LuxR $\mathrm{R}_{\mathrm{WT}}$ 19 and its variants ( $\operatorname{LuxR}_{\mathrm{C} 8-1}$, $\mathrm{LuxR}_{\mathrm{C8}-2}$ and $\left.\mathrm{LuxR}_{\mathrm{N} 86 \mathrm{~K}-\mathrm{C} 245 \mathrm{~W}}\right)$. To perform this manipulation, we 
1 inserted 5'-CATCATCATCATCATCAT-3' immediately downstream of the start codon (ATG)

2 the luxR gene using ExSite PCR.

3 The resultant plasmids were introduced into JW1226 (Plux-gfpuv). The transformants

4 were incubated in $2 \mathrm{ml}$ of LB medium overnight. A $300-\mu \mathrm{l}$ aliquot of the culture was added to

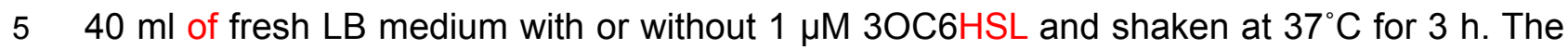

6 cells were subsequently centrifuged at $3,300 \mathrm{rcf}$ for $5 \mathrm{~min}$ to collect the resultant cell pellet.

7 The cells were resuspended to a concentration of $1.2 \times 10^{10}$ cells $/ \mathrm{mL}$ in sonication lysis

8 buffer $(20 \mathrm{mM}$ HEPES, $500 \mathrm{mM} \mathrm{NaCl}, 25 \mathrm{mM}$ imidazole, $5 \%(\mathrm{v} / \mathrm{v})$ glycerol, $0.1 \mathrm{mg} / \mathrm{mL}$

9 lysozyme, and complete EDTA-free protease inhibitor cocktail (Roche Diagnostics,

10 Mannheim, Germany), $\mathrm{pH}$ 7).

11 After chilling on ice for 30 mins, the cell suspension was sonicated on ice, from which we

12 collected $300 \mu \mathrm{L}$ as the lysate. The remainder of the lysate was rigorously centrifuged $(1.7 \times$

$1310^{4} \mathrm{rcf}$ for $30 \mathrm{~min}$ ), and the supernatant was taken as soluble fraction. The total and soluble

14 fraction thus obtained was diluted with $2 \times$ SDS sample buffer ( $0.15 \mathrm{M}$ Tris, $1.2 \%(\mathrm{w} / \mathrm{v}) \mathrm{SDS}$,

$1530 \%(\mathrm{v} / \mathrm{v})$ glycerol, $15 \%(\mathrm{v} / \mathrm{v})$ mercaptoethanol, and $0.18 \mathrm{mg} / \mathrm{L}$ bromophenol blue), and

16 boiled for $5 \mathrm{~min}$. From each sample, $15 \mu \mathrm{L}\left(9 \times 10^{7}\right.$ cells $)$ was loaded on an acrylamide gel

$17(12 \%(w / v))$.

18 The protein in the gel was then transferred to a PVDF membrane for $1 \mathrm{~h}$ at $100 \mathrm{~mA}$ and 19 washed with TBS-T solution (20 mM Tris- $\mathrm{HCL}, 0.8 \% \mathrm{NaCl}$, and $0.2 \%$ Tween 20 ). The 
1 membrane was incubated for $90 \mathrm{~min}$ in $1 \mathrm{~mL}$ of TBS-T containing $5 \%$ skim milk with HRP-

2 HRP-conjugated anti-His-tag antibody (Clontech) at room temperature. The membrane was

3 then washed 3 times in TBS-T and incubated in DAB (Nacalai tesque) solution for 15 min.

4 Finally, the membrane was washed with deionized water for image analysis. 


\section{Results}

\section{LuxR-Plux system for acyl-HSL detection}

3 We adopted a two-plasmid system to evaluate and screen for the function of LuxR and its

4 mutants (Fig. 1A). One plasmid, pTrc-luxR constitutively expressed luxR under the trc

5 promoter (17), and the leaky expression of this promoter was sufficient to support all cellular

6 functions of LuxR. The second plasmid, pAC-Plux-gfpuv encoded gfpuv under the control of

7 the lux promoter. Co-transformation with these two plasmids allowed E. coli cells to

8 fluoresce in response to acyl-HSLs. We found that wild-type LuxR (LuxR $\mathrm{WT}_{\mathrm{WT}}$ ) required 20-25

$9 \mathrm{nM}$ of its cognate signaling molecule $30 \mathrm{C} 6 \mathrm{HSL}$ to achieve a half-maximal level of gene

10 activation $\left(\mathrm{EC}_{50}\right)$ (Fig. 2A). LuxR $\mathrm{R}_{\mathrm{WT}}$ was also activated by acyl-HSLs lacking a 3-oxo group

11 (C6HSL and C8HSL), but this only occurred in the presence of high concentrations of these

12 compounds $\left(\mathrm{EC}_{50}>1 \mu \mathrm{M}\right)$. These results were in good agreement with previous reports

$13(18,19)$.

14 Directed evolution of LuxR for increased sensitivity to C8HSL

15 To isolate LuxR mutants with elevated sensitivity to C8HSLs, we conducted directed

16 evolution (Fig. 1B) using the selection system that we recently developed for the selection

17 of genetic circuits (14).

18 We conducted error-prone PCR mutagenesis (16) on the whole reading frame of LuxR.

19 The resultant PCR product was ligated into pTrc99A (17), grown in liquid medium, and mini- 
1 mini-prepped to yield the library plasmid pTrc-[luxR] (Fig. 1A, square bracket indicates the

2 luxR library). The library plasmid was then co-transformed with pAC-Plux-gfpuv into

3 Approximately $60 \%$ of the clones retained $30 \mathrm{C} 6 \mathrm{HSL}$-responsive fluorescence, whereas

4 others (ca. 40\%) showed a "constitutively-off" phenotype, implying a loss of function/folding

5 capability by deleterious mutations.

6 E. coli was co-transformed with pTrc-[luxR] and pAC-Plux-HSVtk. To isolate LuxR

7 mutants that responded to C8HSL, the pool of JW1226 harboring pTrc-[luxR] was subjected

8 to ON-selection (see experimental section) in the presence of $100 \mathrm{nM} \mathrm{C8HSL}$. The

9 survivors were pooled, from which the mixture of plasmid DNA was isolated for further

10 analysis.

11 C8HSL-sensitive LuXR variants

12 We isolated four LuxR variants with elevated sensitivity to C8HSL (Table 1). The sensitivity

13 to $\mathrm{C} 8 \mathrm{HSL}$ was acquired not by specificity shifting but rather by the overall elevated

14 sensitivity, which was probably due to the elevated transition efficiency from the non-active

15 to active forms. The quantitative liquid-phase characterization revealed that these variants

16 had elevated sensitivity not only to C8HSL but also to all tested acyl-HSLs (the data for

$17 \quad$ LuxR $_{\mathrm{C8}-1}$ and LuxR $\mathrm{C}_{\mathrm{C}-2}$ are shown in Fig. 2B and $\left.\mathrm{C}\right)$.

18 Sequence analysis revealed that all of the four variants shared the same amino acid

19 substitution, C245W, which was the only non-synonymous mutation found in LuxR $R_{\mathrm{C8}-1}$ 
1 1). C245 is positioned at five amino acids from the carboxyl terminus of LuxR. It contains

2 part of a DNA-bonding domain (residues 163-250), which is sufficient to activate gene

3 expression in a ligand-independent manner (20). The residue C245 has never been

4 addressed in relation to the function of LuxR. During the course of alanine-scan

5 mutagenesis of the C-terminal residues (210-250), Trott and Stevens constructed a C245A

6 LuxR mutant and found no significant difference in cellular function compared to wildtype

7 (21). We created a saturation library of LuxR at this position and selected mutants with

8 elevated sensitivity to C8HSL; all of the variants with bright fluorescence had a tryptophan

9 this position. In addition, we created and evaluated LuxR variants with the single mutations

10 C245I, C245L, C245F, and C245Y by using site-directed mutagenesis. None of these

11 mutants were more sensitive to HSLs than to WT, indicating that tryptophan is the only

12 amino acid that confers this high-sensitivity phenotype on LuxR (Fig. S1). Thus, the

13 presence of tryptophan, not the loss of cysteine at this position, is responsible for the

14 elevated sensitivity.

15 In addition to C245W, LuxR $\mathrm{C}_{\mathrm{C}-2}$ possesses four other mutations $(\mathrm{Q} 27 \mathrm{R}, \mathrm{Y} 47 \mathrm{H}, \mathrm{N} 86 \mathrm{~K}$, and

16 I110V) in the signal-binding domain. Of these mutations, we found that N86K alone

17 increased LuxR sensitivity to various acyl-HSLs (Fig. 2D). Structure modeling indicated that

18 N86 is located in the signal-binding domain but is not involved in binding to the acyl-HSLs

19 (Fig. S2). 
Although N86 and C245 are distant in space from each other, the single mutants at these

2 residues ( $L u x R_{N 86 K}$ and $L u x R_{C 8-1}$, respectively) were functionally indistinguishable (Fig. 2B

3 and D). Interestingly, the combination of N86K and C245W resulted in a drastic increase in

4 expression in the absence of signaling molecules (Fig. 2E). This leakiness was markedly

5 suppressed by any of the other mutations (Q27R, Y47H or I110V), recovering the LuxR

6 ON/OFF ratio (Fig. 2C).

8 The effect of C245W on the switching properties of LuxR mutants

9 Located far from the signal-binding domain, C245W appeared to increase the sensitivity of

10 LuxRs without altering their signal preferences. We tested this hypothesis by introducing

$11 \mathrm{C} 245 \mathrm{~W}$ into $\operatorname{LuxR}_{\mathrm{I58N}}$ and $\mathrm{LuXR}_{\mathrm{Y} 62 \mathrm{H}}$, which are specificity-switching mutants that were

12 isolated in different directed evolution programs (they are 30C6HSL-specific and

13 30C8HSL-specific mutants, respectively) (22). As expected, the resultant LuxR $R_{158 \mathrm{~N}-\mathrm{C} 245 \mathrm{~W}}$

14 and LuxR ${ }_{\mathrm{Y} 62 \mathrm{H}-\mathrm{C} 245 \mathrm{~W}}$ showed higher sensitivity than did their respective parents but

15 maintained their original signal preferences (Fig. 3).

16

17 Effective concentration of sensitive mutants

18 To understand how the C245W and M86K elevates the overall sensitivity of LuxR, we fused

19 histidine hexamer tags to the $\mathrm{N}$-termini of the LuxR variants and performed western blotting 
1 of these variants (Fig. 4). Although LuxRs are known to fold better in the presence of acyl-

2 acyl-HSLs (23), the effective cellular concentration of LuxR $R_{W T}$ appeared to be constant,

3 regardless of the presence/absence of $30 \mathrm{C} 6 \mathrm{HSL}$. In contrast, the effective cellular

4 concentrations of mutants, especially for N86K-containing ones (LuxR $R_{C 8-2}$ and LuxR $R_{N 86 K-}$

5 LuxR $\mathrm{N}_{\mathrm{N} 6 \mathrm{~K}-\mathrm{C} 245 \mathrm{~W})}$ were 2.8 times higher with the addition of $3 \mathrm{OC} 6 \mathrm{HSL}$.

6

7 Antagonistic/agonistic response to p-Coumaroyl homoserine lactone.

8 To test the possible antagonist activity of $p$-Coumaroyl homoserine lactone ( $p C \mathrm{HSL})$, we

9 added varying concentrations of $p C H S L$ and a fixed concentration (100 nM) of $30 \mathrm{C} 6 \mathrm{HSL}$ to

10 E. coli harboring LuxR variants with pAC-Plux-gfpuv. In the absence of $p C H S L$, all of the

11 LuxRs were activated by its cognate signal, 30C6HSL. As the concentration of $p C H S L$

12 applied to the system increased, E. coli harboring $\operatorname{LuxR}_{\mathrm{WT}}$ and $\mathrm{LuxR}_{\mathrm{C} 8-1}$ showed a decrease

13 in GFPuv fluorescence (Fig. 5A). Thus, pCHSL acts antagonistically (or in an inhibitory

14 fashion) to LuxR and its variants. In contrast, LuxR $R_{C 8-2}$ was completely free from the

15 antagonistic effect of $p C H S L$ in all tested concentrations $(0-100 \mu \mathrm{M})$ (Fig. 5A).

16 To test the possible agonist function of $p C H S L$ to LuxR variants, we added varying

17 concentrations of $p C H S L$ to $E$. coli expressing LuxR variants with pAC-Plux-gfpuv. Neither

18 LuxR $\mathrm{WT}_{\mathrm{W}}$ nor LuxR $\mathrm{R}_{\mathrm{C}-1}$ responded to $p C H S L$ at any of the tested concentrations (0-100 $\left.\mu \mathrm{M}\right)$. 
1 However, LuxR $\mathrm{Cs}-2$ was activated upon the addition of $p C H S L(>1 \mu \mathrm{M})$ (Fig. 5B). Thus,

$2 p$ CHSL exerts agonist activity on LuxR $\mathrm{R}_{\mathrm{C}-2}$.

\section{Discussion}

\section{Molecular basis of elevation of the signal sensitivity}

6 LuxR requires a high concentration of C8HSL $(>1 \mu \mathrm{M})$ to be activated. Some LuxR

7 homologs, such as CepR from Burkholderia cepacia, are activated by C8HSL with high

8 sensitivity $\left(\mathrm{EC}_{50}<1 \mathrm{nM}\right)(24)$. Collins et al. demonstrated that at least six different amino

9 acid substitutions could independently increase the C8HSL sensitivity of LuxR (8).

10 In the course of reproducing these data with selection-based directed evolution, we found

11 another mutation, C245W, that increased the sensitivity of LuxR variants without changing

12 their signaling preferences (Figs. 2 and 3). Interestingly, none of the previously described

13 C8HSL-sensitizing mutations (such as T33A, S116A, and M135I) (8) were found among the

14 LuxR variants that we isolated. Considering the library size $\left(\sim 10^{6}\right)$ of LuxR that we

15 it is unlikely that none of these mutations existed in the original pool before selection. If the

16 pool actually harbored variants with those mutations, it follows that they were outcompeted

17 by $\mathrm{C} 245$ mutants during the selection process. We do not know the exact mechanism by

18 which this occurred. Notably, we conducted ON-selection in media containing $100 \mathrm{nM}$ of

19 C8HSL, whereas the previous C8HSL-sensitive mutations were found by GFP screening on 
1 a plate containing $200 \mathrm{nM}$ of $\mathrm{C} 8 \mathrm{HSL}$ (8). Indeed, the previous work failed to find any

2 fluorescent clones on the screening plate containing $50 \mathrm{nM} \mathrm{C} 8 \mathrm{HSL}$ (8). Why was C245W

3 found in the previous work? Here again, we do not know exactly. It could possibly be related

4 to the fact that the nucleotide sequence encoding this residue was within the annealing site

5 of PCR primers used for library construction (8). The annealing sites of PCR primers are

6 significantly lower in mutation rates during error-prone PCR (16).

7 Residue 245 is five amino acids upstream from the carboxyl terminus of LuxR. The roles

8 of the LuxR C-terminal domain are DNA binding and recruiting RNA polymerase, and it is

9 not directly involved in signal recognition $(21,25,26)$. Thus, it is unlikely that C245W

10 directly increases the binding affinity of LuxR to C8HSL.

11 We found that the N86K mutation also confers a C245W-like phenotype to LuxR (Fig. 2C

12 and $\mathbf{D}$ ). Because the $\mathrm{N}$-terminal domain inhibits the DNA binding of the $\mathrm{C}$-terminal domain

13 the absence of acyl-HSL (27), it is tempting to hypothesize that both N86 and C245 are

14 deeply involved in this switching machinery. Mutations in these residues may partially

15 the interaction between the domains, thereby facilitating the transition from the inactive form

16 into the active form. It should be noted that all isolated mutants with higher signaling

17 sensitivity showed elevated expression levels, especially in the presence of HSLs (Fig. 4).

18 Because LuxR is more stable and soluble in its active, HSL-bound form (23), C245W

19 probably confers the overall stability of LuxR via elevated efficacy in the transition into the 
1 active soluble form. This "quick-and-dirty" acquisition of signal sensitivity blurred the gap

2 between the inactive and active forms, resulting in a decrease in stringency (Fig. $2 \mathrm{C}$ and

3 LuxR mutants with both the C245W and N86K mutations exhibited increased leaky

4 expression in signal-free conditions and an elevated effective concentration in the absence

5 of HSLs (Fig. 5).

6

7 Single mutation converts LuxR antagonist into agonist

$8 p \mathrm{CHSL}$ is the cognate signal in the quorum sensing system of Rhodopseudomonas

9 palustris (28). However, $p$-Coumaric acid is an antagonist to various other LuxR homologs

10 (29). Many HSLs with aromatic side chains are also antagonists of $\operatorname{LuxR}(30,31)$. We found

11 that $p C H S L$ was indeed an antagonist to LuxR $\mathrm{W}_{W T}$ and its variants (Fig. 5A). However, we

12 also found that it activates $\operatorname{LuxR}_{\mathrm{C} 8-2}$ (Fig. 5B). By creating and testing the single mutants for

13 each of the five mutations found in LuxR $\mathrm{C}_{\mathrm{C}-2}$, we found that $\mathrm{N} 86 \mathrm{~K}$ was responsible for the

14 agonistic response to $p$ CHSL (Fig. S3).

15 It should be noted that LuxR $\mathrm{C} 8-2_{2}$ was obtained in the selection scheme for elevated

16 sensitivity to weak agonist C8HSL, not in the direct selection for an agonistic response to

17 pCHSL. Indeed, LuxR $\mathrm{C} 8-1$, another variant obtained in the same selection, was antagonized

18 by $p C H S L$. Thus, the border between the activator (agonist) and inhibitor (antagonists) is

19 quite blurry, especially in an evolutionary context. If this holds true for other LuxR homologs, 
1 any antagonists of LuxR homologs $(30,32)$ could easily be neutralized or converted into

2 agonists (13) during the non-directional divergence of the receptors, even without counter-

3 counter-selection. This also partially explains why quorum sensing systems can undergo

4 rapid diversification in their signaling sensitivity and exhibit athe frequent emergence of

5 orthogonality and cross-inhibition $(33,34)$.

6

7 Expanding the cellular communication toolbox

8 Synthetic biologists have extensively utilized LuxR family proteins to implement artificial

9 cell-to-cell communications and coordinate the behavior of cell populations $(3,6,7,35)$. To

10 reliably assemble the signaling networks, especially those with complexity, an on-demand

11 but fast-tracked construction of LuxR variants with exact required specifications would be

12 helpful. We found that the mutation C245W increased the general sensitivity of LuxR

13 without affecting its signal preference (Fig. 3). Such "modularity" of mutational effects is

14 desirable because one can quickly and predictably combine them into LuxR with

15 specificities required for various unique contexts.

16 In the laboratory evolution of C8HSL-sensitivity, LuxR acquired mutations that altered its

17 efficiency in the transition to the active form upon the binding of each acyl-HSL, rather than

18 those that directly altered the binding affinity between LuxR and acyl-HSLs. This was

19 achieved by shifting the equilibrium between the inactive/active forms by increasing the 
1 stability of the LuxR ligand-bound (active) state. It has been long recognized that LuxR ( 36 ,

$237)$ and its homologs $(20,38,39,40)$ are stabilized upon binding with HSLs and are

3 protected from proteolytic degradation in the cell. As a result, the effective cellular

4 concentration of active-form LuxR is elevated (Fig. 4). This type of mutation may be one of

5 the most accessible and, therefore, prevailing molecular solutions during the course of

6 divergent evolution LuxR proteins. It would be of interest to determine how and to what

7 extent this type of evolutionary intermediate can diverge and whether it can quickly evolve

8 orthogonality and cross-inhibition, as is frequently seen in nature (33) (34).

9

10

11 Acknowledgments

12 We thank the National BioResource Project (NIG, Japan) for the E. coli KEIO strains. We

13 also thank Dr. Yoichi Mashimo for sequence analysis. This work was partially supported by

14 the Commission for the Development of Artificial Gene Synthesis Technology for Creating

15 Innovative Biomaterial from the Ministry of Economy, Trade and Industry (METI), Japan, the

16 Precursory Research for Embryonic Science and Technology (PRESTO) program of the

17 Japan Science and Technology Agency (JST), Noda Institute for Scientific Research,

18 Futaba Electronics Memorial Foundation, and Shimadzu Science Foundation. 
2

3

4

\section{References}

1. Churchill, M. E. and Chen, L.: Structural basis of acyl-homoserine lactone-dependent signaling, Chem Rev, 111, 68-85 (2011).

2. Steindler, L. and Venturi, V.: Detection of quorum-sensing $\mathrm{N}$-acyl homoserine lactone signal molecules by bacterial biosensors, FEMS Microbiol Lett, 266, 1-9 (2007).

3. Basu, S., Gerchman, Y., Collins, C. H., Arnold, F. H., and Weiss, R.: A synthetic multicellular system for programmed pattern formation, Nature, 434, 1130-1134 (2005).

4. Collins, C. H., Leadbetter, J. R., and Arnold, F. H.: Dual selection enhances the signaling specificity of a variant of the quorum-sensing transcriptional activator LuxR, Nat Biotechnol, 24, 708-712 (2006).

5. You, L., Cox, R. S., 3rd, Weiss, R., and Arnold, F. H.: Programmed population control by cell-cell communication and regulated killing, Nature, 428, 868-871 (2004).

6. Brenner, K., Karig, D. K., Weiss, R., and Arnold, F. H.: Engineered bidirectional communication mediates a consensus in a microbial biofilm consortium, Proc Natl Acad Sci U S A, 104, 17300-17304 (2007).

7. Tamsir, A., Tabor, J. J., and Voigt, C. A.: Robust multicellular computing using genetically encoded NOR gates and chemical 'wires', Nature, 469, 212-215 (2011).

8. Collins, C. H., Arnold, F. H., and Leadbetter, J. R.: Directed evolution of Vibrio fischeri LuxR for increased sensitivity to a broad spectrum of acyl-homoserine lactones, Mol Microbiol, 55, 712-723 (2005).

9. Hawkins, A. C., Arnold, F. H., Stuermer, R., Hauer, B., and Leadbetter, J. R.: Directed evolution of Vibrio fischeri LuxR for improved response to butanoyl-homoserine lactone, Appl Environ Microbiol, 73, 5775-5781 (2007).

10. Fuqua, C., Parsek, M. R., and Greenberg, E. P.: Regulation of gene expression by cell-to-cell communication: acyl-homoserine lactone quorum sensing, Annu Rev Genet, 35, 439-468 (2001).

11. Zhang, R. G., Pappas, K. M., Brace, J. L., Miller, P. C., Oulmassov, T., Molyneaux, J. M., Anderson, J. C., Bashkin, J. K., Winans, S. C., and Joachimiak, A.: Structure of a bacterial quorum-sensing transcription factor complexed with pheromone and DNA, Nature, 417, 971-974

12. (2an)ini, A., Volpari, C., Gargioli, C., Muraglia, E., Cortese, R., De Francesco, R., Neddermann, P., and Marco, S. D.: The crystal structure of the quorum sensing protein TraR bound to its autoinducer and target DNA, EMBO J, 21, 4393-4401 (2002).

13. Koch, B., Liljefors, T., Persson, T., Nielsen, J., Kjelleberg, S., and Givskov, M.: The LuxR receptor: the sites of interaction with quorum-sensing signals and inhibitors, Microbiology, 151, 35893602 (2005).

14. Tashiro, Y., Fukutomi, H., Terakubo, K., Saito, K., and Umeno, D.: A nucleoside kinase as a dual selector for genetic switches and circuits, Nucleic Acids Res, 39, e12 (2011).

15. Baba, T., Ara, T., Hasegawa, M., Takai, Y., Okumura, Y., Baba, M., Datsenko, K. A., Tomita, M., Wanner, B. L., and Mori, H.: Construction of Escherichia coli K-12 in-frame, single-gene knockout 
mutants: the Keio collection, Mol Syst Biol, 2, 20060008 (2006).

16. Cirino, P. C., Mayer, K. M., and Umeno, D.: Generating mutant libraries using error-prone PCR, Methods Mol Biol, 231, 3-9 (2003).

17. Amann, E., Brosius, J., and Ptashne, M.: Vectors bearing a hybrid trp-lac promoter useful for regulated expression of cloned genes in Escherichia coli, Gene, 25, 167-178 (1983).

18. Schaefer, A. L., Hanzelka, B. L., Eberhard, A., and Greenberg, E. P.: Quorum sensing in Vibrio fischeri: probing autoinducer-LuxR interactions with autoinducer analogs, J Bacteriol, 178, 2897-2901 (1996).

19. Winson, M. K., Swift, S., Fish, L., Throup, J. P., Jorgensen, F., Chhabra, S. R., Bycroft, B. W., Williams, P., and Stewart, G. S.: Construction and analysis of luxCDABE-based plasmid sensors for investigating $\mathrm{N}$-acyl homoserine lactone-mediated quorum sensing, FEMS Microbiol Lett, 163, 185192 (1998).

20. Choi, S. H. and Greenberg, E. P.: The C-terminal region of the Vibrio fischeri LuxR protein contains an inducer-independent lux gene activating domain, Proc Natl Acad Sci U S A, 88, 11115-11119

21. (Trom, A. E. and Stevens, A. M.: Amino acid residues in LuxR critical for its mechanism of transcriptional activation during quorum sensing in Vibrio fischeri, J Bacteriol, 183, 387-392 (2001).

22. Tashiro, Y., Kimura, Y., Furubayashi, M., Tanaka, A., Terakubo, K., Saito, K., Kawai-Noma, S., and Umeno, D.: Directed evolution of autoinducer selectivity of Vibrio fischeri LuxR, J Gen Appl Microbiol, in press (2016).

23. Manefield, M., Rasmussen, T. B., Henzter, M., Andersen, J. B., Steinberg, P., Kjelleberg, S., and Givskov, M.: Halogenated furanones inhibit quorum sensing through accelerated LuxR turnover, Microbiology, 148, 1119-1127 (2002).

24. Weingart, C. L., White, C. E., Liu, S., Chai, Y., Cho, H., Tsai, C. S., Wei, Y., Delay, N. R., Gronquist, M. R., Eberhard, A., and Winans, S. C.: Direct binding of the quorum sensing regulator CepR of Burkholderia cenocepacia to two target promoters in vitro, Mol Microbiol, 57, 452-467 (2005).

25. Egland, K. A. and Greenberg, E. P.: Quorum sensing in Vibrio fischeri: analysis of the LuxR DNA binding region by alanine-scanning mutagenesis, J Bacteriol, 183, 382-386 (2001).

26. Finney, A. H., Blick, R. J., Murakami, K., Ishihama, A., and Stevens, A. M.: Role of the C-terminal domain of the alpha subunit of RNA polymerase in LuxR-dependent transcriptional activation of the lux operon during quorum sensing, J Bacteriol, 184, 4520-4528 (2002).

27. Urbanowski, M. L., Lostroh, C. P., and Greenberg, E. P.: Reversible acyl-homoserine lactone binding to purified Vibrio fischeri LuxR protein, J Bacteriol, 186, 631-637 (2004).

28. Schaefer, A. L., Greenberg, E. P., Oliver, C. M., Oda, Y., Huang, J. J., Bittan-Banin, G., Peres, C. M., Schmidt, S., Juhaszova, K., Sufrin, J. R., and Harwood, C. S.: A new class of homoserine lactone quorum-sensing signals, Nature, 454, 595-599 (2008).

29. Bodini, S. F., Manfredini, S., Epp, M., Valentini, S., and Santori, F.: Quorum sensing inhibition activity of garlic extract and p-coumaric acid, Lett Appl Microbiol, 49, 551-555 (2009).

30. Reverchon, S., Chantegrel, B., Deshayes, C., Doutheau, A., and Cotte-Pattat, N.: New synthetic 
analogues of $\mathrm{N}$-acyl homoserine lactones as agonists or antagonists of transcriptional regulators involved in bacterial quorum sensing, Bioorg Med Chem Lett, 12, 1153-1157 (2002).

31. Geske, G. D., O'Neill, J. C., Miller, D. M., Wezeman, R. J., Mattmann, M. E., Lin, Q., and Blackwell, H. E.: Comparative analyses of $\mathrm{N}$-acylated homoserine lactones reveal unique structural features that dictate their ability to activate or inhibit quorum sensing, Chembiochem, 9, 389-400 (20018), K. M., Bu, Y., and Suga, H.: Induction and inhibition of Pseudomonas aeruginosa quorum

33. Elder, A.: Social conflict drives the evolutionary divergence of quorum sensing, Proc Natl Acad Sci U

34. Pollak, S., Omer-Bendori, S., Even-Tov, E., Lipsman, V., Bareia, T., Ben-Zion, I., and Eldar, A.: Facultative cheating supports the coexistence of diverse quorum-sensing alleles, Proc Natl Acad Sci U S A, 113, 2152-2157 (2016).

35. Danino, T., Mondragon-Palomino, O., Tsimring, L., and Hasty, J.: A synchronized quorum of genetic clocks, Nature, 463, 326-330 (2010).

36. Manefield, M., Rasmussen, T. B., Henzter, M., Andersen, J. B., Steinberg, P., Kjelleberg, S., and Givskov, M.: Haloganated furanones inhibit quorum sensing through accelerated LuxR turnover, Microbiology, 148, 1119-1127 (2002).

37. Urbanowski, M. L., Lostroh, C. P., and Greenberg, E. P.: Reversible Acyl-Homoserine Lactone Binding to Purified Vibrio fischeri LuxR Protein, Journal of Bacteriology, 186, 631-637 (2004).

38. Zhu, J. and Winans, S. C.: The quorum-sensing transcriptional regulator TraR requires its cognate signaling ligand for protein folding, protease resistance, and dimerization, Proc Natl Acad Sci U S A, 98, 1507-1512 (2001).

39. Nguyen, Y., Nguyen, N. X., Rogers, J. L., Liao, J., MacMillan, J. B., Jiang, Y., and Sperandio, V.: Structural and mechanistic roles of novel chemical ligands on the SdiA quorum-sensing transcription regulator, MBio, 6, e02429-02414 (2015).

40. Kim, T., Duong, T., Wu, C., Choi, J., Lan, N., Kang, S. W., Lokanath, N. K., Shin, D., Hwang, H.-Y., and Kim, K. K.: Structural insights into the molecular mechanism of Escherichia coli SdiA, a quorumsensing receptor, Acta Crystallogr D Struct Biol, D70, 694-707 (2014). 
1

3 Fig. 1. Directed evolution of LuxR for altered sensitivity. A. Plasmid construction and

B. Genetic selection of LuxR mutants that can respond to

5 C8HSL with improved sensitivity.

6

7 Fig. 2. Activation of Plux-gfpuv with 30C6HSL (filled diamonds), 3OC8HSL (filled

8 circles), C6HSL (open diamonds), and C8HSL (open circles) by LuxR variants. The

9 data were normalized to the optical density and corrected by subtracting the background

10 fluorescence. All measurements were performed in triplicate. The error bars indicate the

11 standard deviation.

13 Fig. 3. The effect of C245W on the sensitivity of LuxR derivatives to different signals.

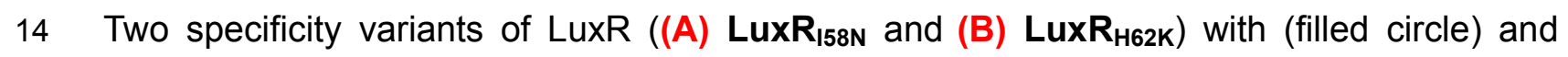

15 without (open circle) $\mathrm{C} 245 \mathrm{~W}$ were constructed and tested their response to $30 \mathrm{C} 6 \mathrm{HSL}$,

$163 \mathrm{OC} 8 \mathrm{HSL}, \mathrm{C} 6 \mathrm{HSL}$, and C8HSL. The data were normalized by the optical density and

17 corrected by subtracting the background fluorescence. The error bars indicate the standard

18 deviation of three independent experiments. 
1 Fig. 4. Western blot analysis of LuxR variants. From cells harboring His-tagged LuxR

2 variants, the total and soluble fractions from approximately $10^{8}$ cells were obtained in the

3 presence $(1 \mu \mathrm{M})$ and absence of $30 \mathrm{OC} 6 \mathrm{HSL}$. The spots were stained with HRP-conjugated

4 anti-His-tag antibody.

6 Fig. 5. Dose response of LuxR variants (filled diamonds: LuxR $R_{W T}$, filled circles:

7 LuxR $\mathbf{R}_{\mathrm{c}-1}$, filled triangles: LuxR ${ }_{\mathrm{c8-2}}$ ) to $p C H S L$. The effect of $p C H S L$ on LuxR variants was

8 studied by measuring the expression level of the reporter ( $g f p_{\mathrm{uv}}$ ) at varying concentrations

9 of $p C H S L$ in the presence of near-saturation concentration $(\mathbf{A}: 100 \mathrm{nM})$ or absence $(\mathbf{B})$ of $1030 \mathrm{OC} 6 \mathrm{HSL}$.

12 Table 1. The LuxR variants found/ used in this work. 
1

2 Table 1. The LuxR variants found/ used in this work.

\begin{tabular}{|c|c|c|}
\hline \multicolumn{2}{|c|}{ Entry number and name } & \multirow{2}{*}{$\begin{array}{l}\text { Mutations } \\
\text { C735G (C245W) }\end{array}$} \\
\hline 1 & LuxR $_{\mathrm{C} 8-1}$ & \\
\hline \multirow[t]{5}{*}{2} & $\operatorname{LuxR}_{\mathrm{C} 8-2}$ & A80G (Q27R) \\
\hline & & T139C (Y47H) \\
\hline & & C258A (N86K) \\
\hline & & A328G (I110V) \\
\hline & & C735G (C245W) \\
\hline \multirow[t]{3}{*}{3} & $\operatorname{LuxR}_{\mathrm{C} 8-3}$ & A545G (D182G) \\
\hline & & C735G (C245W) \\
\hline & & A748G (N250D) \\
\hline \multirow[t]{2}{*}{4} & LuxR c8-4 & A50G (K17M) \\
\hline & & G734T (C245F) \\
\hline
\end{tabular}

3 
Fig 1
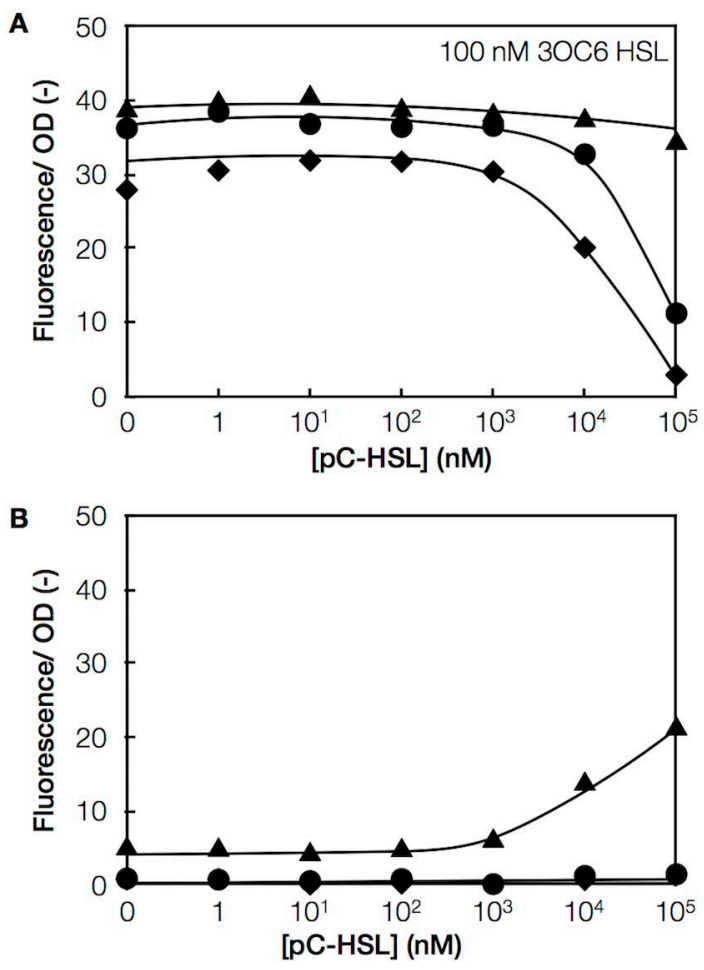
Fig 2

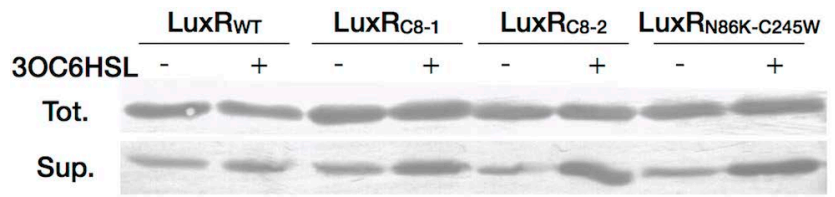


Fig 3

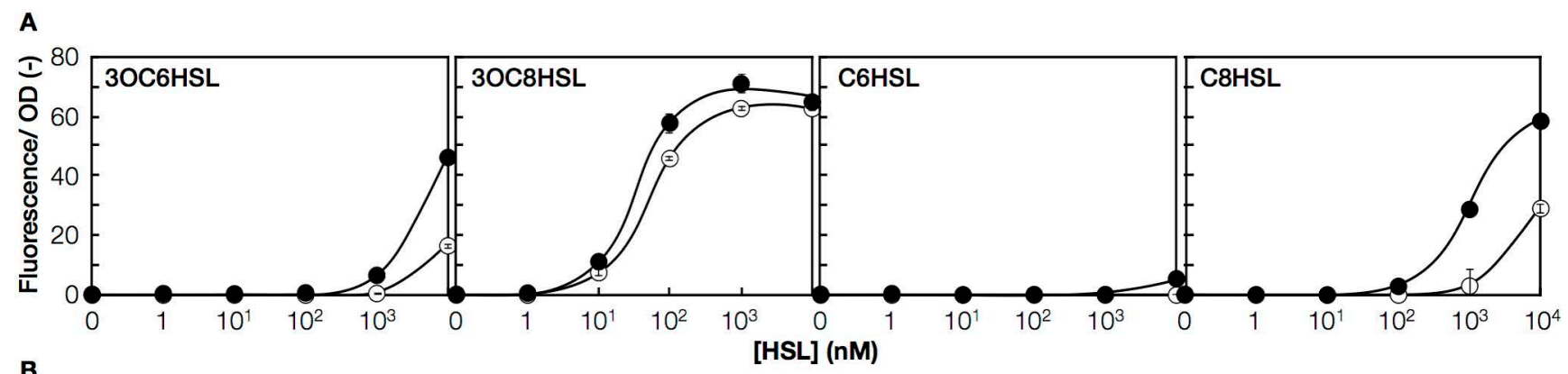

B

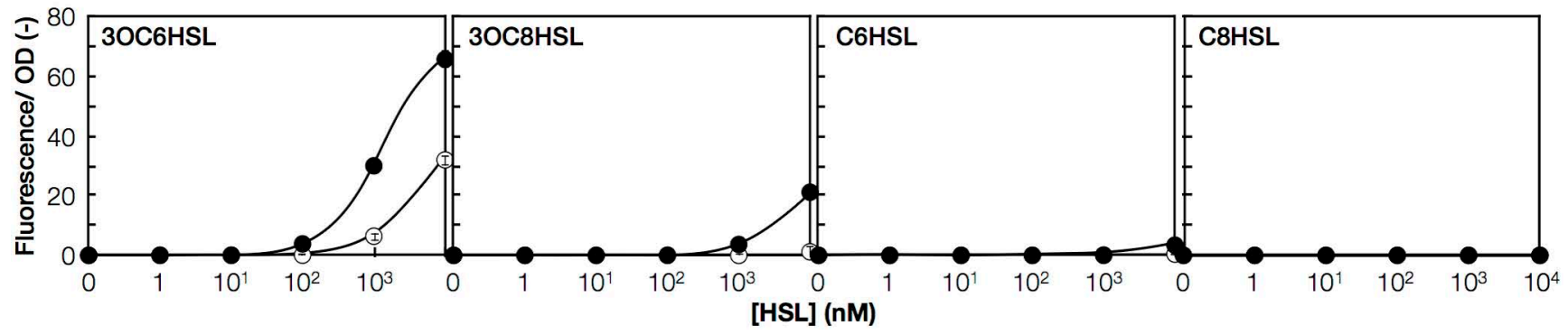


Fig 4

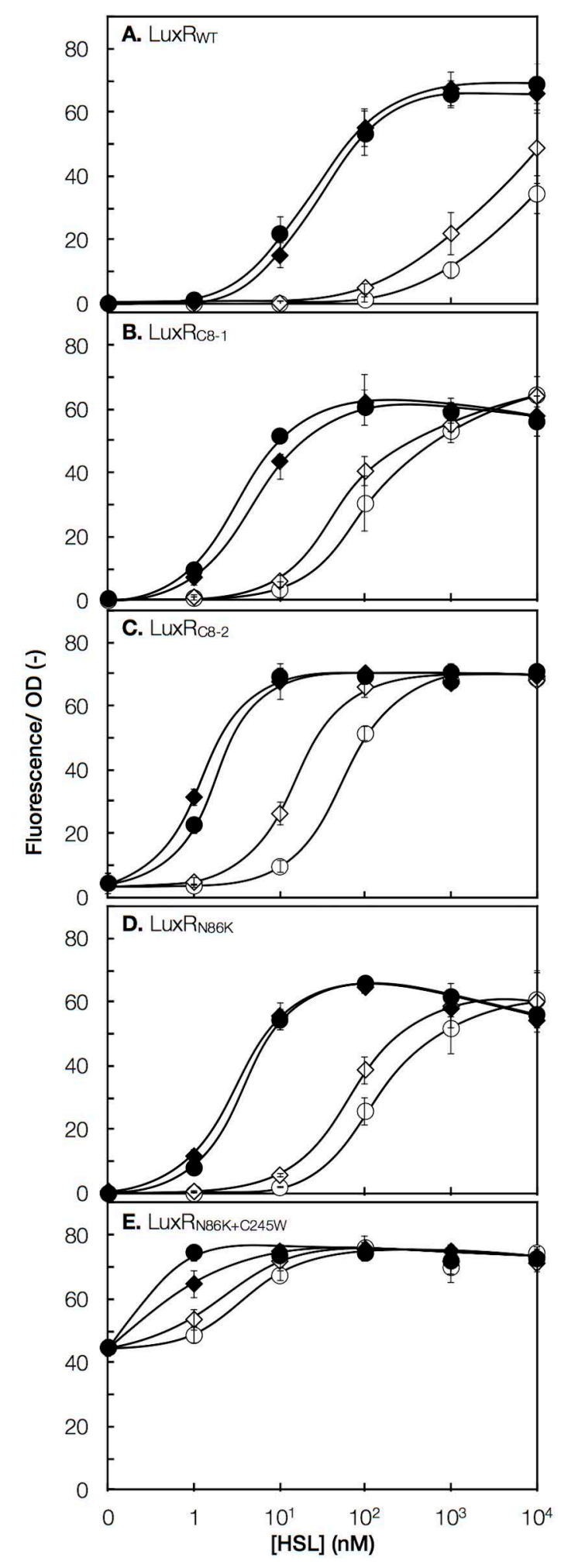


Fig 5

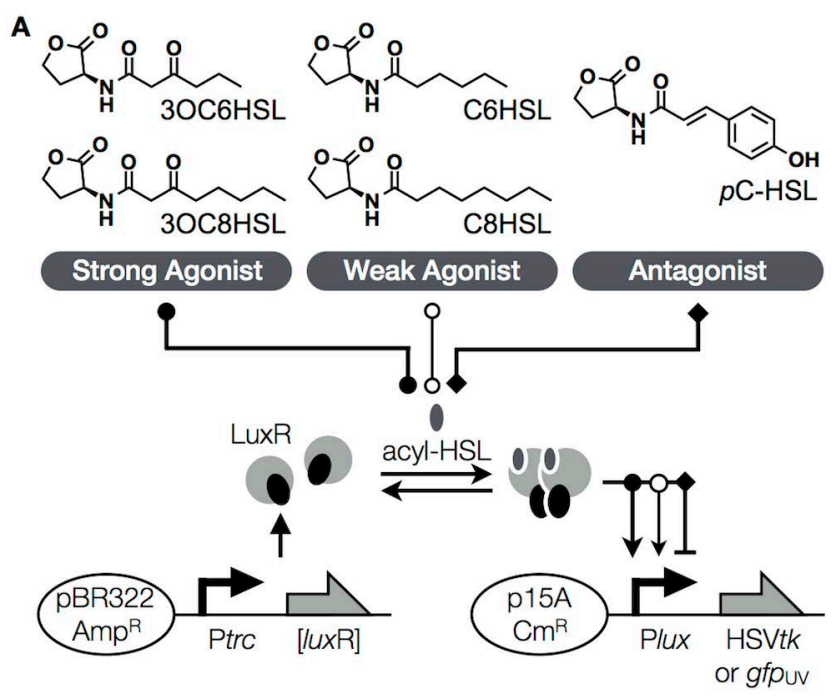

B

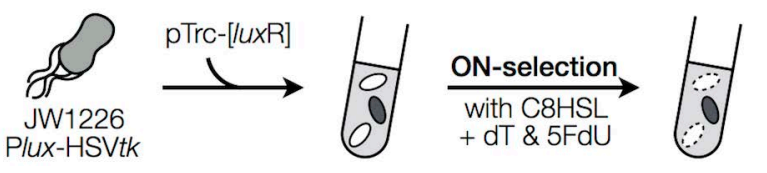

